\title{
SPECTRAL METHODS FOR PERIODIC INITIAL VALUE PROBLEMS WITH NONSMOOTH DATA
}

\author{
PRAVIR K. DUTT AND A. K. SINGH
}

\begin{abstract}
In this paper we consider hyperbolic initial value problems subject to periodic boundary conditions with nonsmooth data. We show that if we filter the data and solve the problem by the Galerkin-Collocation method, recently proposed by us, then we can recover pointwise values with spectral accuracy, provided that the actual solution is piecewise smooth. For this we have to perform a local smoothing of the computed solution.
\end{abstract}

\section{INTRODUCTION}

Spectral methods give very highly accurate approximations to hyperbolic problems with smooth solutions. The naive use of spectral methods on hyperbolic problems with discontinuous solutions, however, produces oscillatory numerical results. The oscillations arising directly from the discontinuity have a Gibbs-like high-frequency character. It has been known for some time that these oscillations are in themselves not insurmountable but contain sufficient information to permit reconstruction of the actual solution. This is achieved by a filtering of the computed values.

A detailed examination of the effect of filtering for linear systems of hyperbolic equations with periodic boundary conditions and discontinuous initial data was made by Majda, McDonnough, and Osher [6]. They showed that for problems in one space dimension it was possible to achieve a convergence rate of infinite order by a proper filtering of the intial conditions and also by applying a filtering during derivative evaluations. However, in two space dimensions this infinite order of accuracy can be obtained only in a domain which excludes the region of influence, and this region spreads linearly with time. Moreover, it is not clear as to how to handle problems where there are discontinuities in the forcing function.

As opposed to global smoothing, one can postprocess the solution obtained by standard Collocation or Galerkin methods by a local smoothing in order to recover spectral accuracy. The idea is based on the observation that while the pointwise convergence of a high-order polynomial approximation to a

Received by the editor June 21, 1991 and, in revised form, June 22, 1992.

1991 Mathematics Subject Classification. Primary 65M06, 65M12, 65M15, 65N35.

Key words and phrases. Discontinuous data, Galerkin-Collocation method, least squares solution, negative Sobolev norms, a priori energy estimates, local smoothing, spectral accuracy.

Research partially supported by CDAC (Centre for Development of Advanced Computing), Pune, India-411007. 
discontinuous solution is very slow, the convergence in a weighted mean is very fast. Local smoothing will be carried out by a convolution in physical space with a localized function and hence by a weighted mean which approximates exceedingly well the exact values of the solution.

From a mathematical point of view, convergence in the mean can be measured in terms of a Sobolev norm of negative order. It can be shown that the error between the computed and exact solution in a negative Sobolev norm decays at a rate which depends only on the order of the norm. The idea was originally developed by Abarbanel, Gottlieb, and Tadmor [1], Gottlieb and Tadmor [5], and Mercier [7]. In their formulation the approximate solution is obtained by first solving a system of ordinary differential equations arising from either the Galerkin or Collocation method, and then postprocessing is applied to the computed solution of this semidiscrete system of equations. We are not aware as to how this procedure would deal with problems in which there are discontinuities in the forcing function also, instead of just in the initial data.

In $[3,4]$ we proposed an alternative formulation for solving hyperbolic partial differential equations which we shall refer to as the Galerkin-Collocation method; this method is spectral in both space and time. In this approach the partial differential equation and initial and boundary conditions are collocated at an overdetermined set of points, and the approximate solution is chosen to be the least squares solution to the overdetermined set of equations thus obtained. It has been proved that for problems with smooth solutions the error decays spectrally in space and time, and computational results for this have been provided in [4]. In this paper we show that for hyperbolic problems with periodic boundary conditions it is possible to recover pointwise values with spectral accuracy using the Galerkin-Collocation method, even when there are discontinuities in the initial data and forcing function, as long as the actual solution is piecewise smooth. In another paper we intend to publish, we hope to extend the theory developed here to general initial-boundary value problems using Legendre and Chebyshev expansions in space and time.

We now outline the contents of this paper. In $\S 2$ we define the Sobolev spaces we shall work in and describe the energy estimates in negative Sobolev norms which are needed in this paper. In $\S 3$ we briefly describe the GalerkinCollocation method and prove that the error between the approximate solution computed by this method and the actual solution in a negative Sobolev norm decays at a rate which depends only on the order of the norm. In $\S 4$ we explain the filtering procedure proposed by Abarbanel, Gottlieb, and Tadmor and show how it can be applied to the approximate solution we obtain by the GalerkinCollocation method to recover pointwise values of the solution with spectral accuracy. Finally in $\S 5$ we present computational results for the proposed method.

\section{ENERGY ESTIMATES FOR HYPERBOLIC INITIAL VALUE PROBLEMS WITH PERIODIC BOUNDARIES}

We consider hyperbolic initial value problems with periodic boundary conditions. Hereafter, $x$ denotes the vector $x=\left(x_{1}, x_{2}, \ldots, x_{d}\right)$.

Let $\Omega=(0,2 \pi)^{d}$ be the space domain and $J=(-1,1)$ be the time interval we are considering. Consider the initial value problem (IVP) 


$$
\begin{gathered}
L u=u_{t}-\sum_{i=1}^{d} A_{i} u_{x_{i}}-B u=F \quad \text { for }(x, t) \in \Omega \times J, \\
u=f \quad \text { for }(x, t) \in \Omega \times\{-1\} .
\end{gathered}
$$

Here, $u$ is a vector-valued function with values in $R^{p}$ and $A_{i}, B$ are matrixvalued functions. Moreover, $A_{i}, B$ are smooth functions of $x$ and $t$ and periodic in $x_{j}$ with period $2 \pi$, for all $j=1, \ldots, d$, and $f$ and $F$ are periodic in each space coordinate with the same period but are not necessarily smooth.

Before we proceed to describe our numerical method and prove its convergence, we need to review some a priori energy estimates which have been proved for solutions of the system $(2.1)$. The interested reader is referred to $[8,9]$ for details.

Let $u$ and $v$ be vector-valued functions of $x$ and $t$ and $2 \pi$-periodic in each space direction. Then we denote

$$
(u, v)_{\Omega \times J}=\iint_{\Omega \times J} u^{*} v d x d t \text { and }\|u\|_{0, \Omega \times J}=\left(\iint_{\Omega \times J}|u|^{2} d x d t\right)^{1 / 2} .
$$

Here, $|u|$ denotes the Euclidean norm of $u$ if $u$ is a vector and $|A|$ denotes the induced matrix norm if $A$ is a matrix. Similarily, we denote

$$
\|u\|_{s, \Omega \times J}=\left(\iint_{\Omega \times J} \sum_{|\alpha|+\beta \leq s}\left|D_{x}^{\alpha} D_{t}^{\beta} u\right|^{2} d x d t\right)^{1 / 2}
$$

where $\alpha=\left(\alpha_{1}, \alpha_{2}, \ldots, \alpha_{d}\right)$ is a multi-index and $D_{x}^{\alpha} u=D_{x_{1}}^{\alpha_{1}} \ldots D_{x_{d}}^{\alpha_{d}} u$. In the same way we define

$$
(u, v)_{\Omega \times\{ \pm 1\}}=\int_{\Omega \times\{ \pm 1\}} u^{*} v d x \text { and }\|u\|_{0, \Omega \times\{ \pm 1\}}=\left(\int_{\Omega \times\{ \pm 1\}}|u|^{2} d x\right)^{1 / 2}
$$

Let

$$
\|u\|_{s, \Omega \times\{ \pm 1\}}=\left(\int_{\Omega \times\{ \pm 1\}} \sum_{|\alpha| \leq s}\left|D_{x}^{\alpha} u\right|^{2} d x\right)^{1 / 2}
$$

where $\alpha$ is a multi-index as above.

We can now state the a priori energy estimates conveniently in terms of the Sobolev norms we have just defined. Let $\psi$ be the solution of the hyperbolic IVP with periodic boundary conditions

$$
\begin{gathered}
L \psi=\phi \text { for }(x, t) \in \Omega \times J, \\
\psi=\theta \text { for }(x, t) \in \Omega \times\{-1\},
\end{gathered}
$$

where $\phi$ and $\theta$ are smooth functions and periodic in space. Then for all integers $s \geq 0$ there exists a constant $C_{s}$, which depends only on the smoothness properties of $A_{i}, B$ such that the estimate

$$
\|\psi\|_{s, \Omega \times J}+\|\psi\|_{s, \Omega \times\{1\}} \leq C_{s}\left(\|\phi\|_{s, \Omega \times J}+\|\theta\|_{s, \Omega \times\{-1\}}\right)
$$


holds. Henceforth we shall use $C$ and $C_{s}$ as generic constants. Next, we need to state a version of (2.3) for negative Sobolev norms.

Let $w$ be a function of $x$ and $t$ which is periodic in space. Let $H=\{\phi: \phi$ is a smooth function of $x$ and $t$ which is periodic in $x$ and has compact support in $t$ \}. We define

$$
\|w\|_{-s, \Omega \times J}=\sup _{\phi \in H} \frac{\left|(w, \phi)_{\Omega \times J}\right|}{\|\phi\|_{s, \Omega \times J}} .
$$

Then $H_{-s, \Omega \times J}$ is defined to be the completion of $H$ with respect to the above norm. Similarily, we define

$$
\|w\|_{-s, \Omega \times\{-1\}}=\sup _{\phi \in H} \frac{\left|(w, \phi)_{\Omega \times\{-1\}}\right|}{\|\phi\|_{s, \Omega \times\{-1\}}} .
$$

With these definitions we can now state the energy estimates in "negative" Sobolev norms. For any $s \geq 0$ there exists a constant $C_{s}$, which depends only on the smoothness properties of $A_{i}, B$, such that

$$
\|\psi\|_{-s, \Omega \times J}+\|\psi\|_{-s, \Omega \times\{1\}} \leq C_{s}\left(\|\phi\|_{-s, \Omega \times J}+\|\theta\|_{-s, \Omega \times\{-1\}}\right),
$$

where $\psi$ is the solution of (2.2), for all $\phi$ and $\theta$. For the sake of completeness we shall provide the proof of (2.4) below, which is very similar to an analogous result proved by Rauch in [8].

We consider the following hyperbolic IVP with periodic boundary conditions:

$$
\begin{gathered}
L^{*} w=-w_{t}+\sum_{i=1}^{d}\left(A_{i}^{T} w\right)_{x_{i}}-B^{T} w=\chi \quad \text { for }(x, t) \in \Omega \times J, \\
w=\mu \text { for }(x, t) \in \Omega \times\{1\},
\end{gathered}
$$

which is the adjoint of (2.2). Notice that for this problem we let time run backwards. The following energy estimate is then valid for the solution $w$ of the adjoint problem:

For every $s \geq 0$ there exists a constant $C_{s}$ which depends only on the smoothness properties of $A_{i}, B$ such that

$$
\|w\|_{s, \Omega \times J}+\|w\|_{s, \Omega \times\{-1\}} \leq C_{s}\left(\|\chi\|_{s, \Omega \times J}+\|\mu\|_{s, \Omega \times\{1\}}\right)
$$

holds.

Let $\psi$ be the solution of (2.2). An integration by parts yields

$$
\left(\psi, L^{*} w\right)_{\Omega \times J}=(L \psi, w)_{\Omega \times J}+(\psi, w)_{\Omega \times\{-1\}}-(\psi, w)_{\Omega \times\{1\}},
$$

since the integrands are periodic in space.

Let $w$ be the solution of the adjoint IVP with periodic boundary conditions

$$
\begin{aligned}
L^{*} w=\chi & \text { for }(x, t) \in \Omega \times J, \\
w=0 & \text { for }(x, t) \in \Omega \times\{1\} .
\end{aligned}
$$

Then by (2.7) we have

(2.9) $\left|(\psi, \chi)_{\Omega \times J}\right| \leq\|L \psi\|_{-s, \Omega \times J} \times\|w\|_{s, \Omega \times J}+\|\psi\|_{-s, \Omega \times\{-1\}} \times\|w\|_{s, \Omega \times\{-1\}}$.

But using the estimate (2.6) we have

$$
\|w\|_{s, \Omega \times J}+\|w\|_{s, \Omega \times\{-1\}} \leq C_{s}\|\chi\|_{s, \Omega \times J},
$$


and this together with (2.9) gives

$$
\left|(\psi, \chi)_{\Omega \times J}\right| \leq C_{s}\left(\|L \psi\|_{-s, \Omega \times J}+\|\psi\|_{-s, \Omega \times\{-1\}}\right) \times\|\chi\|_{s, \Omega \times J} .
$$

Thus, from (2.10) we obtain

$$
\|\psi\|_{-s, \Omega \times J} \leq C_{s}\left(\|L \psi\|_{-s, \Omega \times J}+\|\psi\|_{-s, \Omega \times\{-1\}}\right) .
$$

Next, let $w$ be the solution of the adjoint IVP with periodic boundary conditions

$$
\begin{aligned}
& L^{*} w=0 \text { for }(x, t) \in \Omega \times J, \\
& w=\mu \text { for }(x, t) \in \Omega \times\{1\} .
\end{aligned}
$$

Then (2.7) takes the form

$$
(\psi, \mu)_{\Omega \times\{1\}}=(L \psi, w)_{\Omega \times J}+(\psi, w)_{\Omega \times\{-1\}},
$$

and by (2.6) the estimate

$$
\|w\|_{s, \Omega \times J}+\|w\|_{s, \Omega \times\{-1\}} \leq C_{s}\|\mu\|_{s, \Omega \times\{1\}}
$$

is valid.

Now (2.13) and (2.14) give

$$
\left|(\psi, \mu)_{\Omega \times\{1\}}\right| \leq C_{s}\left(\|L \psi\|_{-s, \Omega \times J}+\|\psi\|_{-s, \Omega \times\{-1\}}\right) \times\|\mu\|_{s, \Omega \times\{1\}},
$$

from which we obtain

$$
\|\psi\|_{-s, \Omega \times\{1\}} \leq C_{s}\left(\|L \psi\|_{-s, \Omega \times J}+\|\psi\|_{-s, \Omega \times\{-1\}}\right) .
$$

Combining (2.11) and (2.15), we get (2.4).

\section{ERROR ESTIMATES FOR BLENDED FOURIER-LEGENDRE METHODS FOR PERIODIC PROBLEMS WITH NONSMOOTH DATA}

Henceforth we shall take $\Omega=(0,2 \pi)$, since the results we state carry over to the general case $\Omega=(0,2 \pi)^{d}$ in a straightforward manner. We now introduce some notation. For each integer $N$ we denote by $\Pi^{N}$ the space of algebraic polynomials in the variable $t$ of degree up to $N$. For each integer $M$ we denote by $S^{M}$ the space

$$
S^{M}=\operatorname{span}\left\{e^{i k x} \mid-M \leq k \leq M\right\} .
$$

Then we define the space $V^{M, N}$ as the tensor product

$$
V^{M, N}=\left\{\phi: \phi(x, t)=\sum_{n=0}^{N} \sum_{m=-M}^{M} a_{m n} e^{i m x} L_{n}(t)\right\},
$$

where $L_{n}(t)$ is the Legendre polynomial of degree $n$. Henceforth we shall assume that there exists a constant $\lambda$ such that

$$
1 / \lambda \leq M / N \leq \lambda \text {. }
$$

For any function $w$ periodic in $x$, which also belongs to $L^{2}(\Omega \times J)$, let $P^{M}, N w$ denote the projection of $w$ into $\left(V^{M, N}\right)^{p}$, i.e.,

$$
P^{M, N} w=\sum_{n=0}^{N} \sum_{m=-M}^{M} w_{m n} e^{i m x} L_{n}(t), \quad \text { where } w=\sum_{n=0}^{\infty} \sum_{m=-\infty}^{\infty} w_{m n} e^{i m x} L_{n}(t)
$$


Henceforth we shall denote $P^{M, N} w$ by $\bar{w}^{M, N}$.

The following results are well known [2, p. 293]. If $w \in H_{k, \Omega \times J}$, then

$$
\left\|w-\bar{w}^{M, N}\right\|_{0, \Omega \times J} \leq C N^{-k}\|w\|_{k, \Omega \times J}
$$

Moreover,

$$
\left\|\bar{w}^{M, N}\right\|_{0, \Omega \times J} \leq\|w\|_{0, \Omega \times J}
$$

Also we have

$$
\left\|w-\bar{w}^{M, N}\right\|_{l, \Omega \times J} \leq C N^{2 l-k}\|w\|_{k, \Omega \times J},
$$

for all $0 \leq l \leq k$. Next, we introduce the norm

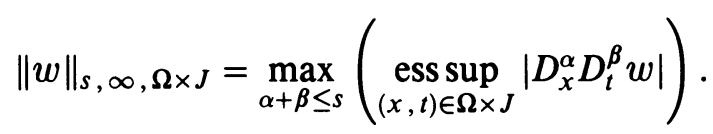

Then we have

$$
\left\|w-\bar{w}^{M, N}\right\|_{l, \infty, \Omega \times J} \leq C N^{2 l-k}\|w\|_{k, \infty, \Omega \times J},
$$

for all $0<l \leq k$.

If $s(x)$ is a periodic function belonging to $L^{2}(\Omega)$, we define

$$
P^{M, 0} s=\sum_{m=-M}^{M} s_{m} e^{i m x}=\bar{s}^{M},
$$

where $s(x)=\sum_{m=-\infty}^{\infty} s_{m} e^{i m x}$.

Similarly, if $h(t) \in L^{2}(J)$, we define

$$
P^{0, N} h=\sum_{n=0}^{N} h_{n} L_{n}(t)=\bar{h}^{N}, \quad \text { where } h(t)=\sum_{n=0}^{\infty} h_{n} L_{n}(t)
$$

We have results similar to (3.1)-(3.3) for the above. Let

$$
\begin{gathered}
\bar{A}^{M-1, N-1}=P^{M-1, N-1} A, \quad \bar{B}^{M-1, N-1}=P^{M-1, N-1} B, \\
\bar{F}^{2 M-1,2 N-1}=P^{2 M-1,2 N-1} F, \quad \bar{f}^{2 M-1}=P^{2 M-1,0} f .
\end{gathered}
$$

We define the differential operator

$$
L^{M, N} w=w_{t}-\bar{A}^{M-1, N-1} w_{x}-\bar{B}^{M-1, N-1} w
$$

We choose as our approximate solution

$$
v^{M, N} \in\left(V^{M, N}\right)^{p}=\left\{\phi: \phi(x, t)=\sum_{n=0}^{N} \sum_{m=-M}^{M} a_{m n} e^{i m x} L_{n}(t), a_{m m} \in R^{p}\right\},
$$

which minimizes

$$
\begin{aligned}
\mathscr{H}^{M, N}\left(w^{M, N}\right)= & \iint_{\Omega \times J}\left|L^{M, N} w^{M, N}-\bar{F}^{2 M-1,2 N-1}\right|^{2} d x d t \\
& +\iint_{\Omega \times\{-1\}}\left|w^{M, N}(x,-1)-\bar{f}^{2 M-1}(x)\right|^{2} d x
\end{aligned}
$$

over all $w^{M, N} \in\left(V^{M, N}\right)^{P}$. The above problem reduces to obtaining a least squares solution to an overdetermined set of equations obtained by collocating 
the modified equation $L^{M, N} w^{M, N}=\bar{F}^{2 M-1,2 N-1}$ and the initial conditions at an overdetermined set of points. We briefly explain this.

Let $\chi_{i}^{M}=\pi i / M, 0 \leq i \leq 2 M-1$, and let $\left\{\tau_{j}^{N}\right\}_{j=0, \ldots, N}$ be the GaussLobatto-Legendre points with $\tau_{0}=-1$ and $\tau_{N}=1$. Notice that

$$
\left(L^{M, N} w^{M, N}-\bar{F}^{2 M-1,2 N-1}\right) \in\left(V^{2 M-1,2 N-1}\right)^{p}
$$

and

$$
\left(w^{M, N}(x,-1)-\bar{f}^{2 M-1}\right) \in\left(S^{2 m-1}\right)^{p} .
$$

Hence we have that

$$
\begin{aligned}
\mathscr{H}^{M, N}\left(w^{M, N}\right)= & \sum_{j=0}^{2 N} \sum_{i=0}^{2 M-1} \alpha_{i j}^{M, N}\left|\left(L^{M, N} w^{M, N}-\bar{F}^{2 M-1,2 N-1}\right)\left(\chi_{i}^{2 M}, \tau_{j}^{2 N}\right)\right|^{2} \\
& +\sum_{i=0}^{4 M-1} \beta_{i}^{M}\left|w^{M, N}\left(\chi_{i}^{2 M},-1\right)-\bar{f}^{2 M-1}\left(\chi_{i}^{2 M}\right)\right|^{2},
\end{aligned}
$$

where $\alpha_{i j}^{M, N}$ and $\beta_{i}^{M}$ are appropriate constants obtained from the GaussLobatto integration formulae. Thus, obtaining a solution to (3.5) is equivalent to solving a least squares problem. It has been shown that if we choose our approximate solution $v^{M, N}$ such that it minimizes the modified functional

$$
\begin{aligned}
\widetilde{\mathscr{H}^{M}, N}\left(w^{M, N}\right)= & \sum_{j=0}^{2 N} \sum_{i=0}^{4 M-1} \alpha_{i j}^{M, N}\left|\left(L w^{M, N}-\bar{F}^{2 M-1,2 N-1}\right)\left(\chi_{i}^{2 M}, \tau_{j}^{2 N}\right)\right|^{2} \\
& +\sum_{i=0}^{4 M-1} \beta_{i}^{M}\left|w^{M, N}\left(\chi_{i}^{2 M},-1\right)-\bar{f}^{2 M-1}\left(\chi_{i}^{2 M}\right)\right|^{2}
\end{aligned}
$$

then we would be committing, in addition, only a spectrally small further error. There is therefore no need to filter the coefficients $A$ and $B$ in practice. The interested reader is referred to [4] for further details. We are interested in another aspect of this minimization procedure. Our approximate solution $v^{M, N}$ is the unique polynomial belonging to $\left(V^{M, N}\right)^{p}$ which satisfies

$$
\begin{aligned}
\iint_{\Omega \times J}\left(L^{M, N} v^{M, N}-\bar{F}^{2 M-1,2 N-1}\right)^{*}\left(L^{M, N} y^{M, N}\right) d x d t & \\
& +\int_{\Omega \times\{-1\}}\left(v^{M, N}-\bar{f}^{2 M-1}\right)^{*} y^{M, N} d x=0,
\end{aligned}
$$

for all $y^{M, N} \in\left(V^{M, N}\right)^{p}$.

We shall now use the above relation to prove that

$$
\left\|L^{M, N} v^{M, N}-\bar{F}^{2 M-1,2 N-1}\right\|_{-s, \Omega \times J} \leq C_{s} N^{1-s}
$$

and

$$
\left\|v^{M, N}-\bar{f}^{2 M-1}\right\|_{-s, \Omega \times\{-1\}} \leq C_{s} N^{1-s},
$$

for any $s>1$. In addition to this, we shall also prove

$$
\left\|L^{M, N} u-\bar{F}^{2 M-1,2 N-1}\right\|_{-s, \Omega \times J} \leq C_{s} N^{-s}
$$


and

$$
\left\|u-\bar{f}^{2 M-1}\right\|_{-s, \Omega \times\{-1\}} \leq C N^{-s} .
$$

With these results established, we can prove Theorem 1 , and the reader is advised to proceed directly to the theorem at the end of this section and continue his perusal of how these results are established only afterwards.

We first need to establish an upper bound on $\mathscr{H}^{M, N}\left(v^{M, N}\right)$. Let $w^{M, N}(x, t)$ $=0$. Then

$$
\begin{aligned}
\mathscr{H}^{M, N}\left(w^{M, N}\right) & \leq\left\|\bar{F}^{2 M-1,2 N-1}\right\|_{0, \Omega \times J}^{2}+\left\|\bar{f}^{2 M-1}\right\|_{\Omega \times\{-1\}}^{2} \\
& \leq\|F\|_{0, \Omega \times J}^{2}+\|f\|_{\Omega \times\{-1\}}^{2},
\end{aligned}
$$

by virtue of (3.2). Hence we can conclude that

$$
\mathscr{H}^{M, N}\left(v^{M, N}\right) \leq C .
$$

To estimate $\left\|L^{M, N} v^{M, N}-\bar{F}^{2 M-1,2 N-1}\right\|_{-s, \Omega \times J}$, we need to bound

$$
\frac{\left|\left(L^{M, N} v^{M, N}-\bar{F}^{2 M-1,2 N-1}, \phi\right)_{\Omega \times J}\right|}{\|\phi\|_{s, \Omega \times J}} \text { for } \phi \in H .
$$

Consider the periodic IVP

$$
\begin{gathered}
L^{M, N} \psi=\phi \text { for }(x, t) \in \Omega \in J, \\
\psi=0 \text { for }(x, t) \in \Omega \times\{-1\} .
\end{gathered}
$$

Then $\psi$ is a smooth function, and using estimate (2.3), we have

$$
\|\psi\|_{s, \Omega \times J} \leq C_{s}\|\phi\|_{s, \Omega \times J},
$$

where $C_{s}$ is a constant which depends only on the smoothness of the coefficients of the modified IVP and hence of the original IVP.

Let $Q^{M, N}$ be the projection operator that maps functions belonging to $H \cap$ $H_{1, \Omega \times J}$ into $V^{M, N}$ defined as:

$$
\begin{aligned}
& Q^{M, N} w \text { is the unique element of } V^{M, N} \text { such that } \\
& \left\|w-Q^{M, N} w\right\|_{1, \Omega \times J}=\inf _{s^{M, N} \in V^{M, N}}\left\|w-s^{M, N}\right\|_{1, \Omega \times J} .
\end{aligned}
$$

Then it is known that

$$
\left\|w-Q^{M, N} w\right\|_{1, \Omega \times J} \leq C N^{1-s}\|w\|_{s, \Omega \times J} .
$$

Let $\tilde{\psi}^{M, N}=Q^{M, N} \psi$. Now

$$
\begin{aligned}
\left(L^{M, N}\right. & \left.v^{M, N}-\bar{F}^{2 M-1,2 N-1}, \phi\right)_{\Omega \times J} \\
= & \left(L^{M, N} v^{M, N}-\bar{F}^{2 M-1,2 N-1}, L^{M, N} \psi\right)_{\Omega \times J} \\
= & \left(L^{M, N} v^{M, N}-\bar{F}^{2 M-1,2 N-1}, L^{M, N} \tilde{\psi}^{M, N}\right) \\
& +\left(L^{M, N} v^{M, N}-\bar{F}^{2 M-1,2 N-1}, L^{M, N}\left(\psi-\tilde{\psi}^{M, N}\right)\right)_{\Omega \times J} .
\end{aligned}
$$

But by (3.7),

$$
\begin{aligned}
& \left(L^{M, N} v^{M, N}-\bar{F}^{2 M-1,2 N-1}, L^{M, N} \tilde{\psi}^{M, N}\right)_{\Omega \times J} \\
& +\left(v^{M, N}-\bar{f}^{2 M-1}, \tilde{\psi}^{M, N}\right)_{\Omega \times\{-1\}}=0 .
\end{aligned}
$$


Now since $\psi=0$ for $(x, t) \in \Omega \times\{-1\}$, we may write

$$
\left(v^{M, N}-\bar{f}^{2 M-1}, \tilde{\psi}^{M, N}\right)_{\Omega \times\{-1\}}=\left(v^{M, N}-\bar{f}^{2 M-1}, \tilde{\psi}^{M, N}-\psi\right)_{\Omega \times\{-1\}} .
$$

Hence we can conclude that

$$
\begin{aligned}
\left(L^{M, N}\right. & \left.v^{M, N}-\bar{F}^{2 M-1,2 N-1}, \phi\right)_{\Omega \times J} \\
= & \left(v^{M, N}-\bar{f}^{2 M-1}, \psi-\tilde{\psi}^{M, N}\right)_{\Omega \times\{-1\}} \\
& \quad+\left(L^{M, N} v^{M, N}-\bar{F}^{2 M-1,2 N-1}, L^{M, N}\left(\psi-\tilde{\psi}^{M, N}\right)\right)_{\Omega \times J} .
\end{aligned}
$$

Now using (3.12), we can conclude that

$$
\left\|L^{M, N}\left(\psi-\tilde{\psi}^{M, N}\right)\right\|_{0, \Omega \times J} \leq C N^{1-s}\|\psi\|_{s, \Omega \times J} .
$$

And applying (3.11), we may write

$$
\left\|L^{M, N}\left(\psi-\tilde{\psi}^{M, N}\right)\right\|_{0, \Omega \times J} \leq C N^{1-s}\|\phi\|_{s, \Omega \times J} .
$$

But

$$
\begin{aligned}
& \left(L^{M, N} v^{M, N}-\bar{F}^{2 M-1,2 N-1}, L^{M, N}\left(\psi-\tilde{\psi}^{M, N}\right)\right)_{\Omega \times J} \mid \\
& \quad \leq\left\|L^{M, N}\left(\psi-\tilde{\psi}^{M, N}\right)\right\|_{0, \Omega \times J} \times\left\|L^{M, N} v^{M, N}-\bar{F}^{2 M-1,2 N-1}\right\|_{0, \Omega \times J} \\
& \quad \leq C_{S} N^{1-s}\|\phi\|_{s, \Omega \times J},
\end{aligned}
$$

by (3.9) and (3.14).

Next, we estimate

$$
\left|\left(v^{M, N}-\bar{f}^{2 M-1}, \tilde{\psi}^{M, N}-\psi\right)_{\Omega \times\{-1\}}\right| .
$$

From (3.9) we have that

$$
\left\|v^{M, N}-\bar{f}^{2 M-1}\right\|_{0, \Omega \times\{-1\}} \leq C .
$$

Now

$$
\left\|\tilde{\psi}^{M, N}-\psi\right\|_{0, \Omega \times\{-1\}} \leq C\left\|\tilde{\psi}^{M, N}-\psi\right\|_{1, \Omega \times J},
$$

by the trace theorem; and so by (3.12) we obtain

$$
\left\|\tilde{\psi}^{M, N}-\psi\right\|_{0, \Omega \times\{-1\}} \leq C N^{1-s}\|\psi\|_{s, \Omega \times J} .
$$

Using estimate (3.11) once again, we conclude that

$$
\left\|\tilde{\psi}^{M, N}-\psi\right\|_{0, \Omega \times\{-1\}} \leq C N^{1-s}\|\phi\|_{s, \Omega \times J} .
$$

Hence, applying (3.16) and (3.17), we get

$$
\left|\left(v^{M, N}-\bar{f}^{2 M-1}, \tilde{\psi}^{M, N}-\psi\right)_{\Omega \times\{-1\}}\right| \leq C_{s} N^{1-s}\|\phi\|_{s, \Omega \times J} .
$$

Combining (3.13), (3.15), and (3.18), we obtain

$$
\left|\left(L^{M, N} v^{M, N}-\bar{F}^{2 M-1,2 N-1}, \phi\right)_{\Omega \times J}\right| \leq C_{s} N^{1-s}\|\phi\|_{s, \Omega \times J},
$$

and this gives us the required estimate

$$
\left\|L^{M, N} v^{M, N}-\bar{F}^{2 M-1,2 N-1}\right\|_{-s, \Omega \times J} \leq C_{s} N^{1-s} .
$$

Next, we estimate

$$
\left\|v^{M, N}-\bar{f}^{2 M-1}\right\|_{-s, \Omega \times\{-1\}} .
$$


Consider the periodic IVP

$$
\begin{gathered}
L^{M, N} \psi=0 \text { for }(x, t) \in \Omega \times J, \\
\psi=\mu \text { for }(x, t) \in \Omega \times\{-1\} .
\end{gathered}
$$

Then $\psi$ is a smooth function, and using estimate (2.3), we have

$$
\|\psi\|_{s, \Omega \times J} \leq C_{s}\|\mu\|_{s, \Omega \times\{-1\}} \text {. }
$$

Let $\tilde{\psi}^{M, N}=Q^{M, N} \psi$. Now

But by (3.7),

$$
\begin{aligned}
\left(v^{M, N}-\bar{f}^{2 M-1}, \mu\right)_{\Omega \times\{-1\}}= & \left(v^{M, N}-\bar{f}^{2 M-1}, \tilde{\psi}^{M, N}\right)_{\Omega \times\{-1\}} \\
& +\left(v^{M, N}-\bar{f}^{2 M-1}, \psi-\tilde{\psi}^{M, N}\right)_{\Omega \times\{-1\}} .
\end{aligned}
$$

$\left(L^{M, N} v^{M, N}-\bar{F}^{2 M-1,2 N-1}, L^{M, N} \tilde{\psi}^{M, N}\right)_{\Omega \times J}+\left(v^{M, N}-\bar{f}^{2 M-1}, \tilde{\psi}^{M, N}\right)_{\Omega \times\{-1\}}=0$.

And since $L^{M, N} \psi=0$ for $(x, t) \in \Omega \times J$, we may write

$$
\begin{aligned}
& \left(L^{M, N} v^{M, N}-\bar{F}^{2 M-1,2 N-1}, L^{M, N} \tilde{\psi}^{M, N}\right)_{\Omega \times J} \\
& \quad=\left(L^{M, N} v^{M, N}-\bar{F}^{2 M-1,2 N-1}, L^{M, N}\left(\tilde{\psi}^{M, N}-\psi\right)\right)_{\Omega \times J} .
\end{aligned}
$$

Hence we can conclude that

$$
\begin{aligned}
\left(v^{M, N}-\bar{f}^{2 M-1}, \mu\right)_{\Omega \times\{-1\}} & \\
= & \left(v^{M, N}-\bar{f}^{2 M-1}, \psi-\tilde{\psi}^{M, N}\right)_{\Omega \times\{-1\}} \\
& \quad+\left(L^{M, N} v^{M, N}-\bar{F}^{2 M-1,2 N-1}, L^{M, N}\left(\psi-\tilde{\psi}^{M, N}\right)\right)_{\Omega \times J} .
\end{aligned}
$$

Thus, we can show

$$
\left\|v^{M, N}-\bar{f}^{2 M-1}\right\|_{-s, \Omega \times\{-1\}} \leq C_{s} N^{1-s},
$$

using (3.22) and the arguments employed earlier.

We now need to estimate

$$
\left\|L^{M, N} u-\bar{F}^{2 M-1,2 N-1}\right\|_{-s, \Omega \times J} .
$$

We know that $u$ satisfies $u_{t}-A u_{x}-B u=F$ in the sense of distributions. Accordingly, we may write

$$
\begin{aligned}
& L^{M, N} u-\bar{F}^{2 M-1,2 N-1} \cdot\left(L^{M, N} u-L u\right)-\left(\bar{F}^{2 M-1,2 N-1}-F\right) \\
& \quad=-\left(\bar{A}^{M-1, N-1}-A\right) u_{x}-\left(\bar{B}^{M-1, N-1}-B\right) u-\left(\bar{F}^{2 M-1,2 N-1}-F\right) .
\end{aligned}
$$

Now by (3.4),

$$
\left\|A-\bar{A}^{M-1, N-1}\right\|_{s, \infty, \Omega \times J} \leq C_{s} N^{-s}\|A\|_{3 s, \infty, \Omega \times J},
$$

and so

$$
\left\|\bar{A}^{M-1, N-1}\right\|_{s, \infty, \Omega \times J} \leq C\|A\|_{3 s, \infty, \Omega \times J},
$$

for $M$ and $N$ large enough. Let us show how to estimate the various terms in (3.24). It is known [2, p. 431] that the projection operator has the property that

$$
\left\|F-\bar{F}^{2 M-1,2 N-1}\right\|_{-s, \Omega \times J} \leq C N^{-s}\|F\|_{0, \Omega \times J} .
$$

Next, we shall estimate $\left\|\left(B-\bar{B}^{M-1, N-1}\right) u\right\|_{-s, \Omega \times J}$. For this we need a lemma. 
Lemma 1. Let $A \in H_{s, \infty, \Omega \times J}$ and $v \in H_{-s, \Omega \times J}$. Then $A v \in H_{-s, \Omega \times J}$, and

$$
\|A v\|_{-s, \Omega \times J} \leq C_{s}\|A\|_{s, \infty, \Omega \times J} \times\|v\|_{-s, \Omega \times J} .
$$

Proof. We have

$$
(A v, \phi)_{\Omega \times J}=\left(v, A^{*} \phi\right)_{\Omega \times J},
$$

by definition. Hence,

$$
\frac{\left|(A v, \phi)_{\Omega \times J}\right|}{\|\phi\|_{s, \Omega \times J}}=\frac{\left|\left(v, A^{*} \phi\right)_{\Omega \times J}\right|}{\|\phi\|_{s, \Omega \times J}}=\frac{\left|\left(v, A^{*} \phi\right)_{\Omega \times J}\right|}{\left\|A^{*} \phi\right\|_{s, \Omega \times J}} \times \frac{\left\|A^{*} \phi\right\|_{s, \Omega \times J}}{\|\phi\|_{s, \Omega \times J}} .
$$

And this gives

$$
\|A v\|_{(-s), \Omega \times J} \leq \sup _{\phi \in H} \frac{\left\|A^{*} \phi\right\|_{s, \Omega \times J}}{\|\phi\|_{s, \Omega \times J}} \times\|v\|_{-s, \Omega \times J} .
$$

Now it is easy to see that

$$
\sup _{\phi \in H} \frac{\left\|A^{*} \phi\right\|_{s, \Omega \times J}}{\|\phi\|_{s, \Omega \times J}} \leq C_{s}\|A\|_{s, \infty, \Omega \times J} .
$$

This gives us the required result.

Thus, we obtain

$$
\left\|\left(B-\bar{B}^{M-1, N-1}\right) u\right\|_{-s, \Omega \times J} \leq C_{s}\left\|B-\bar{B}^{M-1, N-1}\right\|_{s, \infty, \Omega \times J} \times\|u\|_{-s, \Omega \times J} .
$$

But

$\|u\|_{-s, \Omega \times J} \leq\|u\|_{0, \Omega \times J} \quad$ and $\quad\left\|B-\bar{B}^{M-1, N-1}\right\|_{s, \infty, \Omega \times J} \leq C_{s} N^{-s}\|B\|_{3 s, \infty, \Omega \times J}$.

Hence, we obtain

$$
\left\|\left(B-\bar{B}^{M-1, N-1}\right) u\right\|_{s, \infty, \Omega \times J} \leq C_{s} N^{-s} .
$$

Next, we estimate $\left\|u_{x}\right\|_{-s, \Omega \times J}$. Let $\phi \in H$. Then $\left(u_{x}, \phi\right)_{\Omega \times J}=-\left(u, \phi_{x}\right)_{\Omega \times J}$, since both $u$ and $\phi$ are periodic in $x$. Hence,

$$
\frac{\left|\left(u_{x}, \phi\right)_{\Omega \times J}\right|}{\|\phi\|_{s, \Omega \times J}}=\frac{\left|\left(u, \phi_{x}\right)_{\Omega \times J}\right|}{\|\phi\|_{s, \Omega \times J}} .
$$

But $\left\|\phi_{x}\right\|_{(s-1), \Omega \times J} \leq\|\phi\|_{s, \Omega \times J}$. And so we can conclude that

$$
\sup _{\phi \in H} \frac{\left|\left(u_{x}, \phi\right)_{\Omega \times J}\right|}{\|\phi\|_{s, \Omega \times J}} \leq \sup _{\phi \in H} \frac{\left|\left(u, \phi_{x}\right)_{\Omega \times J}\right|}{\left\|\phi_{x}\right\|_{s-1, \Omega \times J}}
$$

which gives us

$$
\left\|u_{x}\right\|_{-s, \Omega \times J} \leq\|u\|_{-s+1, \Omega \times J}
$$

But

$$
\|u\|_{-s+1, \Omega \times J} \leq\|u\|_{0, \Omega \times J} .
$$

And so by the lemma just proved we get

$$
\left\|\left(A-\bar{A}^{M-1, N-1}\right) u_{x}\right\|_{s, \infty, \Omega \times J} \leq C_{s} N^{-s} .
$$

Combining all these estimates, we get the required result

$$
\left\|L^{M, N} u-\bar{F}^{2 M-1,2 N-1}\right\|_{-s, \Omega \times J} \leq C_{s} N^{-s} .
$$

Also we have [2, p. 432]

$$
\left\|u-\bar{f}^{2 M-1}\right\|_{-s, \Omega \times\{-1\}} \leq C N^{-s} .
$$

We can now prove our main theorem. 
Theorem 1. Let $v^{M, N}$ be the solution obtained by minimizing $\mathscr{H}^{M, N}\left(w^{M, N}\right)$ as described in (3.5). Then for all $s \geq 0$ the estimate

$$
\left\|u-v^{M, N}\right\|_{-s, \Omega \times\{1\}}+\left\|u-v^{M, N}\right\|_{-s, \Omega \times J} \leq C_{s} N^{1-s}
$$

holds.

Proof. We have by (3.19) that

$$
\left\|L^{M, N} v^{M, N}-\bar{F}^{2 M-1,2 N-1}\right\|_{-s, \Omega \times J} \leq C_{s} N^{1-s} .
$$

Moreover, by (3.33) we know that

$$
\left\|L^{M, N} u-\bar{F}^{2 M-1,2 N-1}\right\|_{-s, \Omega \times J} \leq C_{s} N^{1-s} .
$$

Using the triangle inequality, we obtain

$$
\left\|L^{M, N}\left(u-v^{M, N}\right)\right\|_{-s, \Omega \times J} \leq C_{s} N^{1-s} .
$$

Finally, we have

$$
\begin{aligned}
\left\|u-v^{M, N}\right\|_{-s, \Omega \times\{-1\}} \leq & \left\|u-\bar{f}^{2 M-1}\right\|_{-s, \Omega \times\{-1\}} \\
& +\left\|\bar{f}^{2 M-1}-v^{M, N}\right\|_{-s, \Omega \times\{-1\}} \\
\leq & C_{s} N^{1-s}
\end{aligned}
$$

using (3.23) and (3.34). Therefore, using estimate (2.4) along with (3.36) and (3.37), we conclude that

$$
\left\|u-v^{M, N}\right\|_{-s, \Omega \times\{1\}}+\left\|u-v^{M, N}\right\|_{-s, \Omega \times J} \leq C_{s} N^{1-s} .
$$

\section{RECOVERING POINTWISE VALUES WITH SPECTRAL ACCURACY}

In this section we briefly describe how the local smoothing proposed by Abarbanel, Gottlieb, and Tadmor can be used to recover pointwise values with spectral accuracy at any point in a neighborhood of which the actual solution is smooth. If we wish to recover the values at $t=1$, the local smoothing is particularly simple. Suppose we wish to obtain the value of the solution at the point $\left(x_{0}, 1\right)$. We assume that there exists a neighborhood

$$
J=\left\{x:\left|x-x_{0}\right| \leq \delta\right\}
$$

in which the actual solution $u(x, t)$ is smooth. Let $\rho(x)$ be a $C_{0}^{\infty}$ function with support in the set $J$ and such that $\rho$ is nonnegative everywhere and $\rho\left(x_{0}\right)=1$. Choose $K=M^{\beta}$ with $0<\beta<1$, and let $D^{K}(\xi)$ denote the Dirichlet kernel

$$
D^{K}(\xi)=\sum_{j=-K}^{K} e^{i j \xi}= \begin{cases}\frac{\sin ((2 K+1) \xi / 2)}{\sin (\xi / 2)}, & \xi \neq 2 m \pi, \\ 2 K+1, & \xi=2 m \pi .\end{cases}
$$

Then, to obtain the regularized version of $v^{M, N}$ at $\left(x_{0}, 1\right)$, we define

$$
R v^{M, N}\left(x_{0}, 1\right)=\frac{1}{2 \pi} \int_{0}^{2 \pi} D^{K}\left(x_{0}-x\right) \rho(x) v^{M, N}(x, 1) d x .
$$

It has been proved in [2, p. 433] that if

$$
\left\|u-v^{M, N}\right\|_{-s, \Omega \times\{1\}} \leq C_{s} M^{-s+1},
$$


then

$$
\left|u\left(x_{0}, 1\right)-R v^{M, N}\left(x_{0}, 1\right)\right| \leq C_{1}(1+\log M) M^{-s+1}+C_{2} M^{-s+1+\beta s},
$$

where the constants $C_{1}$ and $C_{2}$ depend upon the Sobolev norms of $\rho$ and $u$ over the interval $J$. A balance of the errors is achieved by putting $\beta=1 / 2$, in which case we obtain

$$
\left|u\left(x_{0}, 1\right)-R v^{M, N}\left(x_{0}, 1\right)\right|=\mathscr{O}\left(M^{-s / 2+1}\right),
$$

which proves that $u\left(x_{0}, 1\right)$ can be approximated with spectral accuracy starting from the knowledge of the Galerkin-Collocation approximation $v^{M, N}$.

Suppose now that we wish to recover the value of the solution at an interior point $\left(x_{0}, t_{0}\right)$. We assume that $u(x, t)$ is smooth in the set $O$, where

$$
O=\left\{(x, t):\left|x-x_{0}\right| \leq \delta,\left|t-t_{0}\right| \leq \varepsilon\right\} .
$$

Let $\rho(x)$ be a $C_{0}^{\infty}$ function with support in the set $J=\left\{x:\left|x-x_{0}\right|<\delta\right\}$, which is nonnegative everywhere and such that $\rho\left(x_{0}\right)=1$. Similarly, let $\eta(t)$ be a $C_{0}^{\infty}$ function with support in the set $K=\left\{t:\left|t-t_{0}\right|<\varepsilon\right\}$, which is nonnegative everywhere and satisfying $\eta\left(t_{0}\right)=1$. Choose $K=M^{\beta}$ and $L=$ $N^{\gamma}$ with $0<\beta, \gamma<1 / 2$. Let $D^{K}(\xi)$ denote the Dirichlet kernel and $E^{L}\left(\tau, \tau_{0}\right)$ denote the Legendre kernel

$$
E^{L}\left(\tau, \tau_{0}\right)=\sum_{j=0}^{L}(j+1 / 2) L_{j}(\tau) L_{j}\left(\tau_{0}\right)
$$

Then, to obtain the regularized values of $v^{M, N}$ at $\left(x_{0}, t_{0}\right)$, we define

$$
R v^{M, N}\left(x_{0}, 1\right)=\frac{1}{2 \pi} \iint_{\Omega \times J} D^{K}\left(x_{0}-x\right) E^{L}\left(t, t_{0}\right) \rho(x) \eta(t) v^{M, N}(x, t) d x d t .
$$

Once more it can be shown that $R v^{M, N}\left(x_{0}, t_{0}\right)$ approximates $u\left(x_{0}, t_{0}\right)$ with spectral accuracy, and an optimal balance of the errors is obtained by choosing $\beta=\gamma=1 / 3$.

\section{Computational Results}

In this section we demonstrate the efficiency of the method proposed in this paper.

Example 1. Consider the problem

$$
U_{t}-a(x, t) U_{x}-b(x, t) U=F(x, t)
$$

subject to periodic boundary condition $U(0, t)=U(2 \pi, t)$ and initial condition $U(x,-1)=g(x)$. We assume that $g(x)$ has a discontinuity in its derivative.

Case I. Consider

$$
U(x, t)= \begin{cases}(1+t) t+\sin (x), & 0 \leq x \leq \pi, \\ (1+t) t-\sin (x), & \pi \leq x \leq 2 \pi,\end{cases}
$$

and take $a(x, t)=0.5$ and $b(x, t)=0.0$.

Case II. Consider

$$
U(x, t)= \begin{cases}(1+t) \sin (t)+x, & 0 \leq x \leq \pi \\ (1+t) \sin (t)+2 \pi-x, & \pi \leq x \leq 2 \pi\end{cases}
$$

and with the same $a(x, t)$ and $b(x, t)$. 
The results of smoothing the spectral approximation of $U(x, t)$, with $M=$ 128 and $N=17$, are shown in the tables below.

CAse I

\begin{tabular}{|c|c|c|}
\hline $\begin{array}{c}x_{\nu}=\frac{\pi}{8}(\nu+1 / 2) \\
\nu \text { equals }\end{array}$ & $\left|U\left(x_{\nu}, 1\right)-V^{m, n}\left(x_{\nu}, 1\right)\right|$ & $\left|U\left(x_{\nu}, 1\right)-R V^{m, n}\left(x_{\nu}, 1\right)\right|$ \\
\hline 4 & $1.47(-3)$ & $2.69(-8)$ \\
5 & $1.88(-3)$ & $2.28(-8)$ \\
6 & $2.32(-3)$ & $2.67(-8)$ \\
\hline
\end{tabular}

CASE II

\begin{tabular}{|c|c|c|}
\hline $\begin{array}{c}x_{\nu}=\frac{\pi}{8}(\nu+1 / 2) \\
\nu \text { equals }\end{array}$ & $\left|U\left(x_{\nu}, 1\right)-V^{m, n}\left(x_{\nu}, 1\right)\right|$ & $\left|U\left(x_{\nu}, 1\right)-R V^{m, n}\left(x_{\nu}, 1\right)\right|$ \\
\hline 4 & $1.12(-3)$ & $2.88(-8)$ \\
5 & $1.11(-3)$ & $4.19(-8)$ \\
6 & $1.28(-3)$ & $4.48(-8)$ \\
\hline
\end{tabular}

\section{BIBLIOGRAPHY}

1. S. Abarbanel, D. Gottlieb, and E. Tadmor, Spectral methods for discontinuous problems, Numerical Methods for Fluid Dynamics II (K. W. Morton and M. J. Baines, eds.), Oxford University Press, London, 1986, pp. 129-153.

2. C. Canuto, M. Y. Hussaini, A. Quarteroni, and T. A. Zang, Spectral methods in fluid dynamics, Springer, Ser. Comput. Phys., Springer-Verlag, New York, 1988.

3. P. Dutt, Spectral methods for initial-boundary value problems-an alternative approach, SIAM J. Numer. Anal. 27 (1990), 885-903.

4. P. Dutt and A. K. Singh, The Galerkin-collocation method for initial-boundary value problems, J. Comput. Phys. (to appear).

5. D. Gottlieb and E. Tadmor, Recovering pointwise values of discontinuous data within spectral accuracy, Progress and Supercomputing in Computational Fluid Dynamics (E. M. Murman and S. S. Abarbanel, eds.), Birkhäuser, Boston, 1985, pp. 357-375.

6. A. Majda, J. McDonnough, and S. Osher, The Fourier method for nonsmooth initial data, Math. Comp. 32 (1978), pp. 1041-1081.

7. B. Mercier, Analyse numérique des méthodes spectrales, Note CEA-N-2278 (Commissariat a l'Energie Atomique Centre d'Etudes de Limeil, 94190 Villeneuve-Saint Georges).

8. J. Rauch, $\mathscr{L}_{2}$ is a continuable initial condition for Kreiss' mixed problems, Comm. Pure. Appl. Math. 25 (1972), 265-285.

9. M. Taylor, Pseudodifferential operators, Princeton Univ. Press, Princeton, NJ, 1981. INDIA

Department of Mathematics, Indian Institute of Technology at KanPuR, Kanpur, 